\title{
APLICAÇÃO DE UM MODELO DE EQUILÍBRIO ESPACIAL DE PROGRAMAÇÃO QUADRÁTICA PARA QUESTÃO LOGÍSTICA DA SOJA
}

\author{
APPLYING A SPATIAL EQUILIBRIUM MODEL DEVELOPED OF A \\ QUADRATIC PROGRAMMING TO SOYBEAN LOGISTICS
}

\author{
Andréa Leda Ramos de Oliveira Ojima ${ }^{1}$; Akebo Yamakami ${ }^{2}$ \\ ${ }^{1}$ Mestre em Engenharia Elétrica (FEEC/UNICAMP), Pesquisadora do Instituto \\ de Economia Agricola (IEA), andrea@iea.sp.gov.br \\ ${ }^{2}$ Doutor em Engenharia Elétrica (FEEC/UNICAMP), Professor Titular \\ (FEEC/UNICAMP), akebo@dt.fee.unicamp.br
}

Recebido para publicação em: 16/03/2005

Aceito para publicação em 17/07/2005

\section{Resumo}

A proposta deste trabalho é analisar a distribuição logística da soja brasileira aplicando-se um modelo de equilíbrio espacial de programação quadrática. O sistema de transporte da soja é um ponto importante na cadeia produtiva, pois a maior parte dos custos desta commodity é dado pelos custos de transporte. Nesse sentido, a otimização desse segmento é essencial para aumentar a competitividade da soja brasileira no mercado internacional. Neste trabalho foi proposto um cenário $B$, reduzindo-se os custos ferroviários e hidroviários em $20 \%$, já o custo do modal rodoviário foi mantido. Desta maneira, com a aplicação de um modelo de equilíbrio espacial de programação quadrática podemos projetar diferentes cenários, conduzindo políticas para investimentos no setor de transportes.

Palavras-chave: Modelos de equilíbrio especial, Programação quadrática, Transporte e logística

\section{Introdução}

A soja chegou no Brasil com os primeiros imigrantes japoneses em 1908, mas foi introduzida oficialmente no Rio Grande do Sul em 1914. Entretanto a sua expansão se deu efetivamente a partir dos anos 70, com o interesse crescente da indústria de óleo e a demanda do mercado internacional. A criação de novos cultivares pelos melhoristas levou a soja para diversas regiões brasileiras dando estabilidade as áreas de fronteira agrícola do país (OJIMA, 2003).

O complexo soja, isto é grão-farelo-óleo, constitui-se numa das mais importantes commodities nacionais, sendo responsável, na safra 2002/03, pela captação de divisas no mercado 
internacional da ordem de US\$5,0 a US\$6,0 bilhões, de acordo com dados do Ministério do Desenvolvimento, Indústria e Comércio Exterior. A soja movimenta grande montante de recursos e divisas, mas por ser um produto de baixo valor agregado, é necessário que haja uma otimização da produção, estocagem e transporte. Para isso, técnicas de modelagem estão sendo empregadas para tentar reduzir os custos do transporte, tornando-a mais atraente e lucrativa tanto para os produtores como para os investidores (OJIMA, 2003).

O aproveitamento do potencial de expansão da produção de grãos depende do estabelecimento de um sistema eficiente de transporte, nesse sentido Lício (1995) ressalta a relevância da viabilização e integração os corredores de transportes intermodais (rodovia, ferrovia, hidrovia) para aumentar a competitividade dos produtos, integrando as áreas de produção, centros consumidores e o mercado internacional. Segundo Fellin (1993), os modelos de equilíbrio espacial, que utilizam ferramentas de programação quadrática, têm sido usados pela economia agrícola para simular o impacto de novas medidas sobre o setor, além das mudanças nas políticas de transportes.

Assim, a proposta deste trabalho é otimizar a distribuição logística da soja brasileira aplicando-se um modelo de equilíbrio espacial de programação quadrática, sendo a otimização deste segmento essencial para aumentar a competitividade da soja brasileira no mercado internacional.

\section{Sistema de transporte}

Um dos segmentos que mais interfere na eficiência dos diversos setores da economia de um país é o segmento de transporte. No caso brasileiro, em que a maior parte do transporte é feita por rodovias, essa fase da comercialização acaba onerando por demais o bem movimentado. Conforme Wright (1980), o aproveitamento do potencial de expansão da produção de grãos depende do estabelecimento de um sistema eficiente de transporte. Tal sistema terá de comportar volumes maiores a custos menores, permitindo assim que o setor de grãos aumente a sua contribuição no abastecimento interno de alimentos e mantenha sua posição no mercado internacional.

O transporte da soja nas diferentes etapas pode ser realizado por diferentes modais de transporte: rodoviário, ferroviário e hidroviário. No período compreendido entre 1970 a 1999, a modalidade de transporte rodoviário tem absorvido mais da metade dos transportes de cargas no Brasil, sendo em 2000 responsável por $60,4 \%$ contra 20,9\% do transporte ferroviário, 13,9\% do transporte hidroviário e 4,8\% de outros (duto e aéreo). Conforme Caixeta Filho (1996:04), “essa predominância do modo rodoviário pode ser explicada pelas dificuldades que outras categorias de transporte enfrentam para atender eficientemente aos aumentos de demanda em áreas mais afastadas 
do País, as quais não são servidas por ferrovias ou hidrovias".

A distribuição do transporte de carga pelos diferentes modais é decorrência da geografia de cada país e também dos incentivos governamentais no setor. A participação dos modais rodoviário, ferroviário e hidroviário no transporte de cargas brasileiro é substancialmente diferente daquela encontrada em outros países de dimensões continentais similares. Em geral Países com pequena extensão territorial vocacionam o seu transporte no modal rodoviário, enquanto que países com grandes extensões, com exceção do Brasil, priorizam o transporte para os modais ferroviário e hidroviário, dado que estes modais possuem uma maior eficiência e competitividade no transporte de longas distâncias (OJIMA, 2004).

A infra-estrutura de transporte disponível no Brasil é significativamente menor do que aquela existente em diversos países em desenvolvimento e de grandes extensões territoriais. No Gráfico 1 é apresentado a densidade de transporte por modal, este índice é calculado a partir do número de quilômetros de infra-estrutura disponível por cada $\mathrm{km}^{2}$ de área do País. No Gráfico 1 , a densidade é calculada para cada $1000 \mathrm{~km}^{2}$ de área do País.

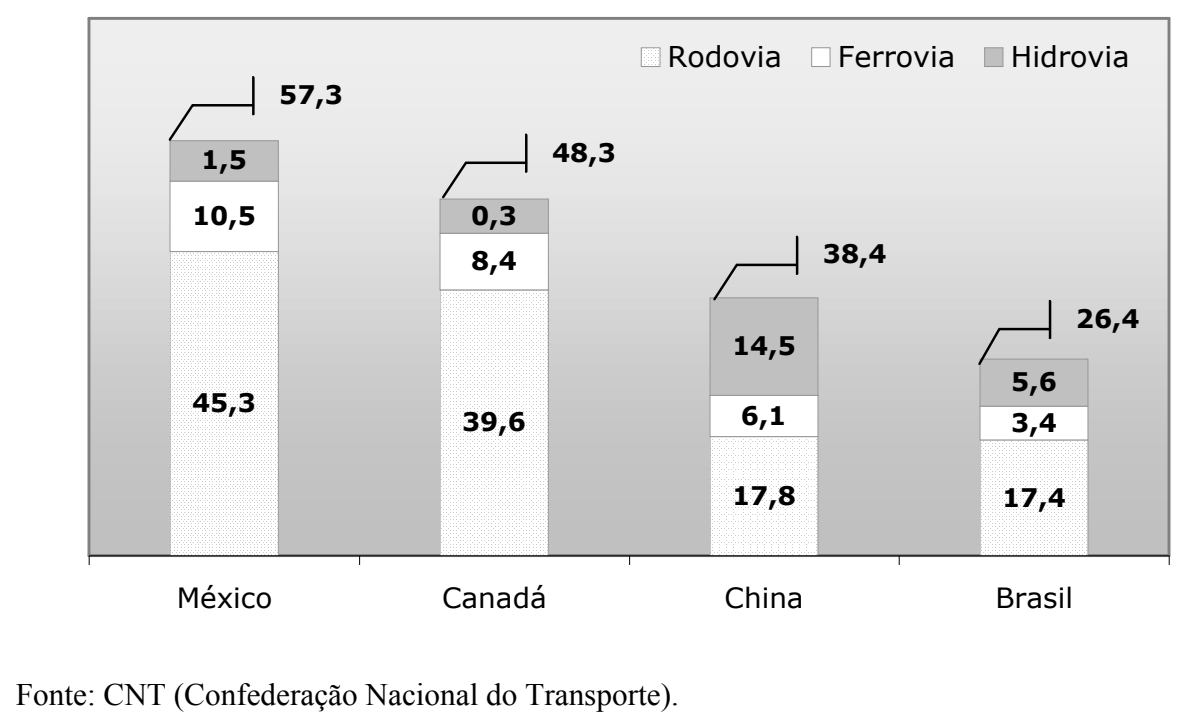

Gráfico 1 - Densidade de Infra-estrutura de Transporte $\left(\mathrm{km} / 1000 \mathrm{~km}^{2}\right)$

Conforme Lieb (1978), as diferentes características entre os modais, como custos e outros aspectos qualitativos, pode ser economicamente desejável que entre a origem e o destino de um determinado produto sejam utilizados mais que uma modalidade de transporte, utilizando as vantagens inerentes a cada uma delas, o que resulta num serviço de menor custo e/ou de melhor qualidade.

A complementação entre as modalidades de transporte envolvidas num sistema intermodal implicará atividades de transbordo, isto é, recursos humanos e equipamentos para transferir as 
mercadorias de um meio de transporte para outro. Assim a intermodalidade, além de nortear os investimentos no setor dos transportes, contribui para redução dos custos, uma vez que os custos com transporte ferroviário e hidroviário são menores que os custos rodoviários, ocorrendo uma tendência de se substituir o transporte rodoviário de longa distância por transportes alternativos, que deve implicar no aumento da competitividade da soja no mercado internacional de grãos, assim como também a sua participação nas exportações mundiais.

Enquanto isso, o transporte da soja esbarra na estrutura apresentada pelas estradas do País, gerando perdas do produto e também, devido ao desgaste sofrido pelo caminhão, um aumento do preço do frete. Segundo Puzzi (1986), no Brasil um dos componentes que mais pesam no custo final dos grãos é o frete, devido à falta de hidrovias, à insuficiência de ferrovias e à precariedade de estradas pavimentadas.

\section{O modelo}

O uso da programação não linear é comum em modelos econômicos que envolvem preços endógenos. Produção e/ou consumo usualmente ocorrem em regiões separadas espacialmente, cada uma delas possui uma relação de oferta e demanda. Se os preços entre as regiões forem dados em maior grau pelos custos de transporte inter-regionais, a comercialização ocorrerá e será direcionada por tais custos. Modelando esta situação coloca-se pelos menos algumas questões a serem respondidas, entre elas, quem irá produzir e consumir, em que quantidades e em que níveis a comercialização se dará (McCARL and SPREEN, 2001).

As condições de equilíbrio espacial para commodities com custos de transporte ligados às regiões de exportação e importação podem ser vistas no Gráfico 2, onde P corresponde ao preço e Q a quantidade. A quantidade da commodity negociada é igual ao excesso de oferta (ef) na região de exportação, enquanto a quantidade importada é igual ao excesso de demanda (gh) no preço de equilíbrio, assumindo que o custo de transporte é igual a zero entre as duas regiões. Com a existência de tarifas e custos de transporte medidos pela distância vertical entre as curvas de importação demandada e a exportação ofertada (ab), a diferença do preço entre as regiões de importação e exportação é igual às tarifas e custos de transporte. Estes custos e tarifas são compartilhados pelas regiões de exportação e importação, de acordo com as respectivas elasticidades de cada região. 


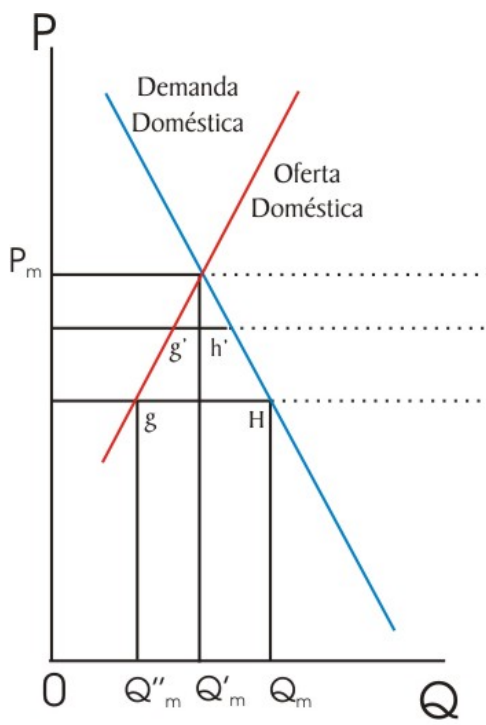

Região Importadora

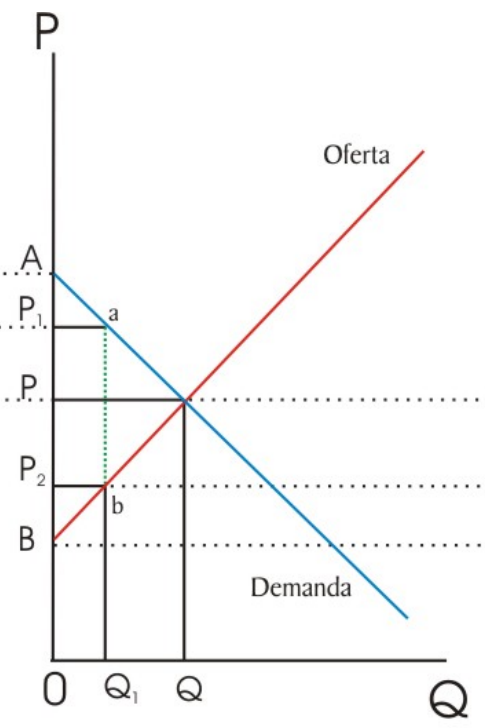

Mercado Internacional

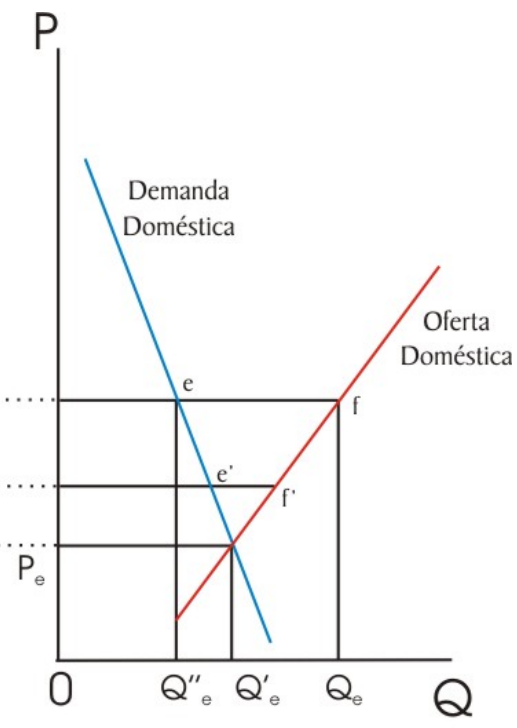

Região Exportadora

Fonte: Fellin, 1993:38.

Gráfico 2 - Equilíbrio Internacional de Comercialização entre duas Regiões

No Gráfico 2 as tarifas e custos de transporte $(\mathrm{ab})$ aumentam o preço na região importadora de $\mathrm{P}$ para $\mathrm{P} 1$, assim o aumento do preço pago pela região importadora resulta em um decréscimo na quantidade comercializada de Q para Q1. A proporção do diferencial do preço pago pelos produtores das regiões exportadoras (P2) e a incorporação para os consumidores na importação (P1) podem ser calculados com a função das elasticidades de oferta e demanda.

A estrutura teórica deste modelo pode ser expandida, incluindo regiões multi-exportadoras e importadoras, transporte multimodal e multi-commodity. Os problemas de equilíbrio espacial são expressos matematicamente, através da maximização das áreas sob as curvas de demanda menos as áreas sob as curvas de oferta e menos os custos de transporte.Por exemplo, $i$ regiões de comércio substituem duas regiões descritas na Figura 1, fazendo com que a demanda na região $i$ seja dada por:

$\mathrm{p}_{\mathrm{i}}=\mathrm{d}_{\mathrm{i}}\left(\mathrm{y}_{\mathrm{i}}\right)$

onde,

$\mathrm{p}_{\mathrm{i}}=$ preço de demanda na região $\mathrm{i}$

$\mathrm{y}_{\mathrm{i}}=$ quantidade demandada com preço $\mathrm{p}_{\mathrm{i}}$ na região $\mathrm{i}$

A função de oferta para região é definida por:

$p^{i}=s_{i}\left(z_{i}\right)$

onde,

$p^{i}=$ preço de oferta na região $i$

$\mathrm{Z}_{\mathrm{i}}=$ quantidade ofertada com preço $\mathrm{p}^{\mathrm{i}}$ na região $\mathrm{i}$

Assumindo que a inclinação da curva de demanda é descendente e que a inclinação da curva de oferta é ascendente, temos que:

$$
\begin{aligned}
& \partial\left(\mathrm{d}_{\mathrm{i}}\left(\mathrm{y}_{\mathrm{i}}\right)\right) / \mathrm{y}_{\mathrm{i}}<0 \\
& \mathrm{e} \\
& \partial\left(\mathrm{s}_{\mathrm{i}}\left(\mathrm{z}_{\mathrm{i}}\right)\right) / \mathrm{z}_{\mathrm{i}} \geq 0
\end{aligned}
$$


A função lucro é definida como a área abaixo da curva da demanda menos integral abaixo da curva de oferta.

A função lucro individual pode ser expressa matematicamente por:

$\mathrm{W}_{\mathrm{i}}\left(\mathrm{z}_{\mathrm{i}}, \mathrm{y}_{\mathrm{i}}\right)=\int^{\mathrm{yi}} \mathrm{d}_{\mathrm{i}}\left(\eta_{\mathrm{i}}\right) \mathrm{d} \eta_{\mathrm{i}}-\int^{\mathrm{zi}} \mathrm{s}_{\mathrm{i}}\left(\in_{\mathrm{i}}\right) \mathrm{d} \in_{\mathrm{i}}$,

O total ou função lucro é:

$\mathrm{W}=\sum_{\mathrm{i}} \mathrm{W}_{\mathrm{i}}\left(\mathrm{z}_{\mathrm{i}}, \mathrm{y}_{\mathrm{i}}\right)$

Se $\mathrm{x}_{\mathrm{ij}}$ representa o total de movimentações aceitáveis de $i$ para $j$ com um custo de transporte $\mathrm{t}_{\mathrm{ij}}$, então a expressão para lucro poder ser descrita como:

$$
\mathrm{NW}=\mathrm{W}-\sum_{\mathrm{i}} \sum_{\mathrm{j}} \mathrm{t}_{\mathrm{ij}} \mathrm{x}_{\mathrm{ij}}
$$

Para que o chamado equilíbrio espacial seja observado, requer-se que os fluxos direcionados à região $j$ sejam maiores ou iguais à demanda da região $j$, então:

$$
\mathrm{y}_{\mathrm{j}}<\sum_{\mathrm{i}} \mathrm{x}_{\mathrm{ij}} \text {, para todo } \mathrm{j}
$$

e que os fluxos originados na região $i$ não sejam maiores que as quantidades ofertadas pela região $i$, então:

$$
\mathrm{z}_{\mathrm{i}} \geq \sum_{\mathrm{j}} \mathrm{x}_{\mathrm{ij}} \text {, para todo } \mathrm{i}
$$

O modelo matemático que determina o nível de produção e consumo em cada região, o preço de comercialização em cada região e a quantidade comercializada entre as regiões pode então ser representada por:

$$
\operatorname{Max} N W=\sum_{i} W_{i}\left(z_{i}, y_{i}\right)-\sum_{i} \sum_{j} t_{i j} x_{i j}
$$

Sujeito a

$\mathrm{y}_{\mathrm{j}}<\sum_{\mathrm{i}} \mathrm{x}_{\mathrm{ij}}$ para todo $\mathrm{j}$

$\mathrm{z}_{\mathrm{i}} \geq \sum_{\mathrm{j}} \mathrm{x}_{\mathrm{ij}}$ para todo $\mathrm{i}$

$\mathrm{y}_{\mathrm{j}}, \mathrm{z}_{\mathrm{i}}, \mathrm{x}_{\mathrm{ij}} \geq 0$

Quando as funções de oferta e demanda são lineares, i.e.,

$\mathrm{p}_{\mathrm{i}}=\mathrm{a}_{\mathrm{i}}-\mathrm{b}_{\mathrm{i}} \mathrm{y}_{\mathrm{i}}$

$p^{i}=c_{i}+d_{i} z_{i}$

O problema será igual:

$$
\begin{aligned}
& \operatorname{Max} \Sigma_{i}\left(a_{i} y_{i}-1 / 2 b_{i} y_{i}^{2}-c_{i} z_{i}-1 / 2 d_{i} z_{i}^{2}\right)-\Sigma_{i} \Sigma_{j} t_{i j} x_{i j} \\
& y_{j}<\Sigma_{i} x_{i j} \text { para todo } j, \\
& z_{i} \geq \Sigma_{j} x_{i j} \text { para todo } i, e \\
& y_{j}, z_{i}, x_{i j} \geq 0 .
\end{aligned}
$$

O termo quadrático incluído na função objetivo (3.16) é a razão do grau de não-linearidade do modelo proposto. 
A solução sugerida pelo modelo determina o fluxo de soja-grão das regiões de oferta para as regiões de demanda doméstica, portos e demanda internacional. No modelo inicialmente foram identificadas as regiões de oferta e demanda de soja.

A escolha das regiões que compõe o modelo partiu de uma Análise de Mercado da Commodity Soja, considerando também a infra-estrutura disponível. Neste estudo foi analisado o comportamento dos últimos anos das variáveis: produção de soja, rendimento médio, área cultivada, exportações e capacidade instalada de processamento (planta industrial).

A escolha dos Estados que compõem o modelo partiu da tentativa de caracterização do mercado, considerando as regiões que tiveram um desenvolvimento mais predominante nos últimos anos e o potencial de expansão baseado nas fronteiras agrícolas em constante crescimento.

Para caracterização das regiões de excesso de oferta e demanda partiu-se da seguinte premissa: se a produção de soja for maior que a quantidade processada, essa região fica caracterizada como uma região de excesso de oferta, caso contrário essa região fica caracterizada como uma região de excesso de demanda. Para os estado do Paraná, Mato Grosso e Mato Grosso do Sul foram identificadas diferentes microrregiões, tanto com relação a produção como ao processamento.

O processamento das informações para o modelo de equilíbrio espacial desenvolvido para a movimentação de soja no Brasil foi feito utilizando-se o software General Algebraic Modeling System - GAMS e a programação matemática adotada foi a não-linear (função objetivo não-linear e restrições lineares). O solver utilizado neste caso (MINOS) é uma junção do método do Gradiente reduzido com o método quasi-Newton.

O objetivo do modelo de equilíbrio é maximizar a função lucro total definida por Samuelson (1952) usando a estrutura básica proposta por Takayma e Judge (1971).

Os dados que compõe o modelo são: produção, consumo, preços de comercialização do mercado nacional e internacional, fretes dos diferentes modais, tiveram como base o ano de 2002. Os dados de produção tiveram como fonte o Instituto Brasileiro de Geografia e Estatística (IBGE) e o Departamento de Agricultura dos Estados Unidos (USDA), enquanto que consumo teve como base a Associação Brasileira de Industrias de Óleos Vegetais (ABIOVE). Os dados de elasticidadespreço de oferta e demanda foram baseadas nos estudos desenvolvidos por Fuller, 2000.

Os custos de transporte dos modais rodoviário e ferroviário no modelo foram estimados através de equações lineares baseadas nas distâncias entre os pontos de carregamento e os de recepção (origem/destino). O comportamento do custo dos modais (variável de resposta) foi analisado através de um modelo de regressão linear múltipla, utilizando um banco de dados de fretes praticados em 2002, com base nos dados do Sistema de Informação de Fretes (SIFRECA), em todo o território brasileiro de acordo com a distância e os diferentes meses do ano. Já, o modal 
hidroviário apresentou um comportamento regular durante o ano com preços constantes para as distâncias envolvidas.

Deste modo, para o modal rodoviário partiu-se da hipótese de existir um comportamento diferenciado para fretes com distâncias de até 500 quilômetros e com uma sazonalidade de preços para o primeiro e segundo semestre do ano (variáveis explicativas). Assim, para o modal rodoviário temos:

$$
\begin{aligned}
& Y=X \beta+\varepsilon \\
& y=\beta_{0}+\beta_{1}(\mathrm{~km}) \delta_{1}+\beta_{2}(\mathrm{~km})\left(1-\delta_{1}\right)+\beta_{3} \delta_{1}+\beta_{4} \delta_{2}+\varepsilon \\
& \delta_{1}=\left\{\begin{array}{l}
1 \text { se distância } \geq 500 \\
0 \text { se distância }<500
\end{array} \quad \delta_{2}=\left\{\begin{array}{l}
0 \text { se semestre }=1 \\
1 \text { se semestre }=2
\end{array}\right.\right.
\end{aligned}
$$

O modelo linear de custo foi implementado em um programa estatístico Minitab 13.0. Para o transporte ferroviário as equações foram desenvolvidas da mesma maneira que para o modal rodoviário, não incluindo a variável semestre, visto que a esta variável explicativa não obteve significância estatística quando inserida no modelo e foi observado um comportamento linear dos preços ferroviários durante o ano, a distância referência encontrada foi de $850 \mathrm{~km}$.

\section{Resultados e discussão}

O modelo de equilíbrio espacial obtido para a movimentação da soja brasileira passou por um processo de verificação e validação dos dados para determinação da sua confiabilidade e utilidade, podendo assim gerar cenários futuros. O primeiro passo desenvolvido foi a verificação dos dados resultantes, analisando-se os fluxos gerados entre a oferta e a demanda de soja nas respectivas regiões e nos dois semestres envolvidos, com ênfase nas quantidades comercializadas e preços de oferta.

$\mathrm{Na}$ Tabela 1 podemos verificar que as quantidades ofertadas e demandas pelo modelo foram equivalentes.

Tabela 1 - Oferta e Demanda de Soja

\begin{tabular}{lll|ll}
\hline Oferta & Volume (mil ton) & Preço (US\$/ton) & Demanda & Volume (mil ton) \\
\hline BA & 158,4 & 159,0 & MS-CN & 241,7 \\
RO & 363,3 & 157,4 & MT-CS & 253,6 \\
MA & 573,8 & 169,8 & MT-SD & 292,8 \\
PR-CO & $1.514,8$ & 173,0 & PR-NC & 347,4 \\
MS-SO & $1.667,7$ & 161,8 & AM & 523,2 \\
PR-SO & $1.682,4$ & 172,4 & SP & $1.358,1$ \\
PR-O & $1.941,8$ & 172,4 & PR-SD & $1.473,8$ \\
MG & $2.720,4$ & 172,0 & Sub-Total & $\mathbf{4 . 4 9 0 , 6}$ \\
MT-NO & $3.637,1$ & 150,5 & JAPÃO & $2.793,3$ \\
GO & $3.662,4$ & 162,7 & CHINA & $8.914,3$ \\
RS & $3.951,6$ & 173,8 & EUROPA & $10.529,9$
\end{tabular}




\begin{tabular}{lllll}
\cline { 3 - 4 } MT-N & $4.854,4$ & 150,0 & Sub-Total & $\mathbf{2 2 . 2 3 7 , 5}$ \\
\hline Total & $\mathbf{2 6 . 7 2 8 , 1}$ & - & Total & $\mathbf{2 6 . 7 2 8 , 1}$ \\
\hline PR-SO: Sudoeste PR-O: Oeste PR-CO: Centro-oeste PR-SD: Sudeste PR-NC: Centro-norte & \\
MT-N: Norte MT-NO: Noroeste MT-SD: Sudeste MT-CS: Centro-sul MS-SO: Sudoeste MS-CN: Centro
\end{tabular}

Segundo Fellin (1993:79), os procedimentos de validação de modelos podem variar e são necessários para interpretar e analisar o comportamento dos resultados do modelo. Assim, os dados gerados pelo modelo foram analisados através de estatísticas de correlação entre os dados reais e o modelo.

A Tabela 2 mostra os fluxos obtidos, o modal rodoviário foi vocacionado para o abastecimento interno e interligação dos modais, que em geral ocorrem para distâncias inferiores a $850 \mathrm{~km}$, sendo um modal complementar ao ferroviário e hidroviário que são utilizados para distâncias superiores, já os modais ferroviário e hidroviário foram ativados para o transporte de longas distâncias com destino ao mercado internacional.

Tabela 2 - Fluxo de Movimentação de Soja Obtidas pelo Modelo

\begin{tabular}{|c|c|c|c|c|}
\hline ORIGEM & DESTINO 1 & \multicolumn{3}{|c|}{ DESTINO 2} \\
\hline Oferta & Demanda & Transbordo & Porto & Demanda Inter \\
\hline $\begin{array}{c}\text { RO } \\
\text { MT-NO }\end{array}$ & $\mathrm{AM}(\mathrm{r})$ & P. Velho (h) & Itacoatiara $(\mathrm{h})$ & China e Japão (m) \\
\hline $\begin{array}{l}\text { BA } \\
\text { MA }\end{array}$ & & $\begin{array}{l}\text { Araguari (f) } \\
\text { Estreito (f) }\end{array}$ & $\begin{array}{l}\text { Vitória (f) } \\
\text { Itaqui (f) }\end{array}$ & China (m) \\
\hline MS-SO & $\begin{array}{c}\text { MS-CN (r) } \\
\text { SP (r) } \\
\text { PR-NC (r) }\end{array}$ & Londrina (f) & Paranaguá (f) & China (m) \\
\hline PR-O & & & Paranaguá (f) & China (m) \\
\hline PR-CO & PR-NC (f) & & Paranaguá (f) & China (m) \\
\hline PR-SO & PR-SD (f) & & Paranaguá (f) & China (m) \\
\hline MT-N & $\begin{array}{l}\text { MT-SD (r) } \\
\text { MT-CS (r) }\end{array}$ & A. Araguaia (f) & Santos (f) & Europa (m) \\
\hline GO & $\begin{array}{l}\text { MT-SD (r) } \\
\text { SP (r) }\end{array}$ & Goiânia (f) & Santos (f) & Europa (m) \\
\hline MG & SP (r) & & Santos (f) & Europa $(\mathrm{m})$ \\
\hline $\mathrm{RS}$ & & & Rio Grande (f) & Europa, China e Japão (m) \\
\hline
\end{tabular}

Um novo cenário (B) foi proposto alterando-se sensivelmente os custos de transporte ferroviário e hidroviário em $20 \%$ e o rodoviário mantido. Os fluxos mantiveram-se com um aumento nos níveis de volume transportados pelos modais ferroviário e hidroviário, 5,3\% e 3,2\% respectivamente, em decorrência da redução dos custos, foi verificada também alteração nos preços de equilíbrio.

Segundo Ojima, 2004 as políticas para o setor de transportes devem ser direcionadas para incentivar a intermodalidade, visto que os modais viários são interdependentes e cada um deles tem 
uma determinada vocação tendo vantagens e desvantagens com relação às distâncias percorridas, o volume transportado e o transit time (tempo percorrido entre origem e destino).

Como visto anteriormente a matriz e a densidade de infra-estrutura de transporte brasileira não é equivalente a situação dos países de mesma extensão territorial, assim redistribuir de maneira eficiente o transporte de carga no Brasil pode potencializar a sua inserção no mercado internacional. Assim, existe a possibilidade de utilização do modelo para testar diferentes hipóteses, considerando as melhorias nos sistemas de transporte que podem acarretar em uma redução dos custos com frete envolvidos. Ou seja, podemos incluir em um cenário hipotético uma nova rota de escoamento da produção com a viabilização de um trecho hidroviário ou ferroviário para verificar o seu impacto no contexto geral.

Por exemplo, hoje temos dois exemplos importantes nesta direção. O primeiro é implantação da hidrovia Tocantins-Araguaia, planejada para o transporte da região Centro-Oeste para o Porto de Belém (PA) ou ligando a região até o terminal hidroviário Porto Franco (MA) e seguindo por ferrovia até o Porto de Itaqui (MA). O segundo seria o prolongamento da Ferronorte, ferrovia que interliga o estado do Mato Grosso ao Porto de Santos, até o município de Rondonópolis (MT). Ambos são bons exemplos do esforço que o governo federal tem feito para o setor de transportes brasileiro. Tais investimentos estão em fase embrionária, mas apontam para uma política preocupada em fomentar uma condição mais favorável.

Uma plena adequação do sistema não depende apenas de uma malha viária disponível e em condições de uso, mas também da estrutura complementar, como armazéns e terminais ferroviário, hidroviário e marítimo. A estrutura teórica deste modelo pode ser expandida, incluindo regiões multi-exportadoras e importadoras e multi-commodity. A introdução de novos dados ao modelo, incluindo mais regiões de excesso de oferta e demanda, portos, demanda internacional e novos fluxos de transporte poderão gerar resultados que mais se aproxime dos valores ótimos que possam garantir uma maior inserção do Brasil no mercado internacional da commodity soja.

\begin{abstract}
The purpose of this paper is to analyze the logistic distribution of the Brazilian soybean applying a spatial equilibrium model developed of a quadratic programming. The soybean transportation system is an important point in the productive chain, the most part of the costs of this commodity is given by transportation costs. And, in this manner, optimize this sector is essential to increase the competitiveness of the Brazilian soybean at the international market. In this paper a frame B was considered and reduced the railroad and water road costs in $20 \%$, already the truck road cost was kept. This way, we have reached a model that can project new frames by switching the transportation costs and to conduce the policy makers to new investments in the sector.
\end{abstract}

Key words: Soybean transportation, spatial equilibrium model, transportation and logistics 


\section{Referências}

CAIXETA FILHO, J. V. Transporte e logística no sistema agroindustrial. Preços Agrícolas: Mercados Agropecuários e Agribusiness, v. 10, n. 119, p. 2-7, set. 1996.

CONFEDERAÇÃO NACIONAL DO TRANSPORTE. Disponível em: http://www.cnt.org.br. Acesso em: fev. 2003.

FELLIN, L. R. International corn and soybean transportation system: quadratic programming models. $134 \mathrm{f}$. 1993. Tese (Doutorado em Economia Agrícola), Texas A\&M University, College Station.

FULLER, S. Effect of improving South American transportation system on U.S. and Soutth American corn and soybean economies. U.S. Department of Agriculture, Agricultural Marketing Service, 2000. 40 p.

LÍCIO, A. Os eixos estruturadores e dos corredores de transportes. Revista de Política Agrícola, Brasília, v. 9, n. 4, p. 3-4, 1995.

LIEB, R. C. Transportation: the domestic system. Reston: Reston Publ. Co., 1978. cap.7.

McCARL, B. A.; SPREEN, T. H. Applied mathematical programming using algebraic systems. Texas A\&M University. College Stations, Texas, 2001. [mimeo].

OJIMA, A. L. R. O. Analysis of the logistical movement and competitiveness of soybean in the brazilian center-north region: an application of a spatial equilibrium model with quadratic programming. In: INTERNATIONAL CONFERENCE ON AGRI-FOOD CHAIN/NETWORKS ECONOMICS AND MANAGEMENT, 4., Ribeirão Preto. Anais... Ribeirão Preto: USP, 2003. p. 24.

Análise da movimentação logística e competitividade da soja brasileira: uma aplicação de um modelo de equilíbrio espacial de programação quadrática. 79 f. 2004. Dissertação (Mestrado), Universidade Estadual de Campinas, Faculdade de Engenharia Elétrica e de Computação. Campinas - SP.

PUZZI, D. Abastecimento e armazenagem de grãos. Campinas: Instituto Campineiro de Ensino Agrícola, 1986. p. 81-90.

SAMUELSON, P. A. Spatial Price Equilibrium and Linear Program. American Economic Review, v. 42, p. 283-303, 1952.

SOARES, M. G.; GALVANI, P. R. C.; CAIXETA FILHO, J. V. Transporte de soja em grãos e farelo de soja no Brasil. Preços Agrícolas: Mercados Agropecuários e Agribusiness, v. 11, n. 126, p. 26-29, abr. 1997.

TAKAYAMA, T.; JUDGE, G. G. Spatial and Temporal Price and Allocation Models. Amsterdan: North Holland Publ. Co., 1971.

WRIGHT, C. L. Análise econômica de transporte e armazenagem de grãos: estudo do corredor de exportação de Paranaguá. Brasília: GEIPOT, 1980. 187 p. 